\title{
A Parallel Computing Algorithm for Moving Targets Tracking in Wireless Sensor Networks
}

\author{
XIONG Jing ${ }^{\text {a }}$, LIU Zhi-jing ${ }^{b}$ and TANG Guo-liang ${ }^{c}$ \\ School of Computer Science and Technology, Xidian University, Xi'an 710071, China \\ axiongjing mail@163.com, ${ }^{\mathrm{b}}$ liuzhijing@vip.163.com, ${ }^{\mathrm{c}}$ Datang110@126.com
}

\begin{abstract}
Keywords: Wireless Sensor Networks (WSN); Collaborative tracking; Feature extraction; Dynamic clustering algorithm; Parallel computing.
\end{abstract}

Abstract. In this paper, we address the problem of moving target feature extraction and collaborative tracking in wireless sensor networks (WSN), and present a parallel computing algorithm for moving human collaborative tracking. At first, WSN optimization deployment: divide the monitors in WSN into the two types: Behavior Recognition Monitor (BRM) and Collaborative Tracking Monitor (CTM), and settle all the monitors utilizing FCM algorithm into many groups. Secondly, parallel detection and behavior recognition to get the collaborative tracking target. Finally, a multi-points feature extraction scheme for WSN monitors to track the suspicious target collaboratively. We also compare our algorithm with three existing solutions, the statistics result shows that our scheme has a better detection accuracy and tracking performance.

\section{Introduction}

Wireless Sensor Networks (WSN) consists of a large number of low cost sensor nodes that have various information collection functions. Due to the low distance and scene change limitation, and extensive applications such as target tracking, infrastructure monitoring, habitat sensing, and battlefield surveillance [1], the research of WSN pays more and more attentions to researchers. However, the calculated amount and energy consumption are unavoidable problems related to WSN research for its limitation that inconvenience power supply and computation burden for single node [3].

Collaborative tracking is a typical application for wireless sensor networks, which could be used to intelligent safe guard system in civil and flight target collaborative location for radar system in military. As for moving human collaborative tracking, the tracking accuracy could not be promised but the extract feature extraction and matching. However, the schemes in feature extraction at present are not suit for WSN nodes for its computation burden, and the superiority of WSN is not be exploited. On the one hand, the schemes based on networks structure on the research of collaborative tracking are popular [6], such as, a quantized measurement fusion framework focus on the energy and bandwidth scheduling strategy is proposed by Yan Zhou[2], it gets a efficient energy scheme on collaborative tracking. Moreover, a dynamic cluster member selection method for multi-target tracking in WSN [4] that good for multi-target collaborative tracking. However, all the schemes are not consider the feature extraction and matching and it is not suit for human collaborative tracking. On the other hand, the moving target feature extraction for tracking present a lot of achievement, such as Yuan Yuan proposed a visual object tracking based on backward model validation method [5] that is good for single point tracking, but the complicate model building work is a problem for WSN node and the superiority of WSN is not be utilized.

To overcome the problems above, we propose a parallel computing scheduling for moving human collaborative tracking in WSN. At first, divide the sensor node into two types: Behavior Recognition Monitor (BRM) and Collaborative Tracking Monitor (CTM), and use BRM are clustering origin points to settle all the monitors utilizing FCM algorithm into many groups. Secondly, parallel detection and behavior recognition to get the collaborative tracking target. Finally, a multi-points feature extraction scheme for WSN monitors to collaborative track the suspicious target. In the experiment part, we compare our algorithm with three existing solutions, the statistics result shows that our scheme has a better detection accuracy and tracking performance. 


\section{Monitor nodes optimization deployment}

For collaborative tracking with suspicious moving targets, the monitor nodes in WSN could be divided into two types: the behavior recognition monitors (BRM) and the collaborative tracking monitors (CTM). BRM recognize the behavior of moving people to select the suspicious target and extract features of it, then CTM focus on the feature match and collaborative tracking the target. On the original step, the system we discussed utilize the dynamic clustering algorithm to optimization deploy all the monitor nodes in WSN, the result refers to Fig 1 is that to set the cluster centers are BRM with a number of CTM deploy around.

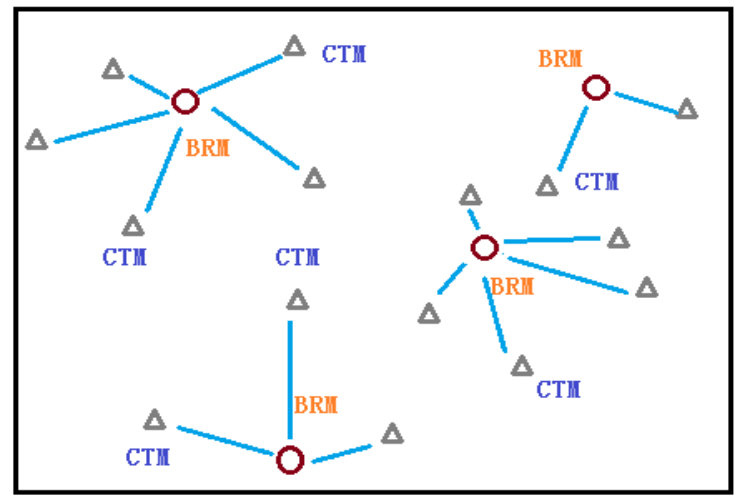

Fig 1. The monitor nodes deployment result

In our system, a dynamic clustering algorithm based on FCM algorithm is proposed which original clustering centre is confirmed, and we could get the groups consist of BRM and CTM, each monitor node complete the behavior recognition and relay tracking task in the same group.

Although FCM algorithm is good for fast clustering and could get the better clustering accuracy, it easy fall into terminal point, so the choosing for original point is important. Considering the features of our system, let BRM as the original clustering centre. We assume $X=\left\{x_{1}, x_{2}, \ldots, x_{n}\right\}$ as the total monitor nodes set, divide the $\mathrm{X}$ into $\mathrm{c}$ groups $(2 \leq \mathrm{c} \leq \mathrm{n})$, there are $\mathrm{c}$ number of $\mathrm{BRM}$ as original clustering centre $V=\left\{v_{1}, v_{2}, \ldots, v_{c}\right\}$ at the same time. The degree of membership for each monitor node could be described as fuzzy matrix $U=\left[u_{i j}\right], u_{i j}$ is the degree of membership between the monitor $\mathrm{i}$ and $\mathrm{j}$, that must satisfy the conditions $\sum_{i=1}^{c} u_{i j}=1$ and $u_{i j} \in[0,1]$. The destination function could be described as: $J\left(U, v_{1}, \ldots, v_{c}\right)=\sum_{i=1}^{c} J_{i}=\sum_{i=1}^{c} \sum_{j}^{n} u_{i j}^{m} d_{i j}^{2}$

In the equation above, $v_{i}$ is the clustering centre in the fuzzy group $\mathrm{i}, d_{i j}=\left\|v_{i}-x_{j}\right\|$ is the Euclidian Distance between the i clustering centre and the j monitor node [7]. $M^{m \in[1, \infty)}$ is the fuzzy weighting index that benefit for noise restraining and making membership function smoothing. Two conditions contribute to get the lowest value for destination function mentioned above [8]: 


$$
v_{i}=\frac{\sum_{j=1}^{n} u_{i j}^{m} x_{j}}{\sum_{j=1}^{n} u_{i j}^{m}}
$$

The degree of membership m controls the share degree of fuzzy groups, it also play an important role in noise restrain and effect the concave-convex of destination function. Considering the convergence of the algorithm, the value area of $\mathrm{m}$ is related with sampling amount $\mathrm{X}$, the best value is in the range [1.3, 2.5].

Though the algorithm that set the confirmed original clustering point, we can get the cluster result which BRM are the cluster centre points. In each group according to the result includes a BRM and several CTM, CTM receives the tracking command from BRM in the same group, and product a dynamic monitor subnet. When the CTM in the boundary of a group is busy, the WSN will dynamic cluster for expanding the radius of the busy subnet according to a clustering intervene factor $\beta$ to share responsibility for busy subnet.

\section{Moving targets behavior recognition and parallel computing}

After monitors in WSN have been deployed, each node should be prepared to detect moving targets. In this paper, we proposed an improved algorithm to detection moving people that combine Mix Gaussian Model (MGM) with area fusion method. The moving target detection result could see in Fig 2 below:

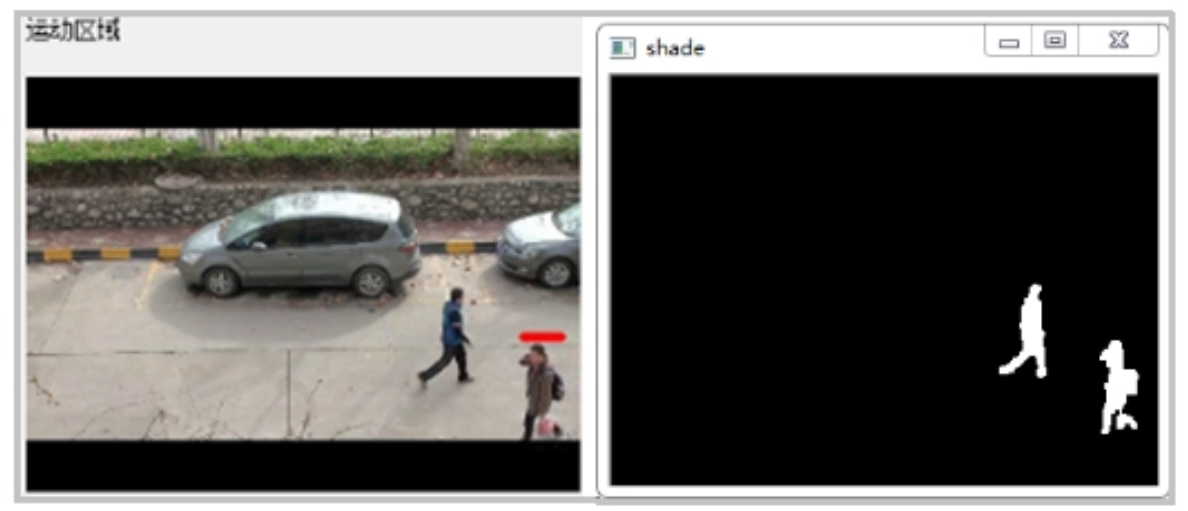

( a ) The original frame image (b ) Moving target detection result

Fig 2. An example of moving target detection

The background in an original frame image just like (a) in Fig2 always insist of interference, such as tree branch swaying, fallen leaves and moving area shelter from. Moving target detection using traditional method could not get rid of these noises, but use the improved algorithm that combine MGM with area fusion algorithm a better result could be got. In Fig2, (b) is the moving area detection result.

Not all the moving targets need to be collaborative tracked, so BRM should recognize the behaviors of targets and focus on the suspious one. Each monitor in the WSN share the same behavior library which some behavior templates would be defined, such as walking, running, jumping, etc. Surveillance system can definite some regular behaviors in the beginning, so other behaviors are suspicious.For reducing the computation pressure of BRM and promote the work efficiency, a parallel computing algorithm to be proposed based on DOT module[9]. The DOT includes three layers: D-layer, O-layer and T-layer, so the composition of the parallel computing module could be describe as Fig 3: 


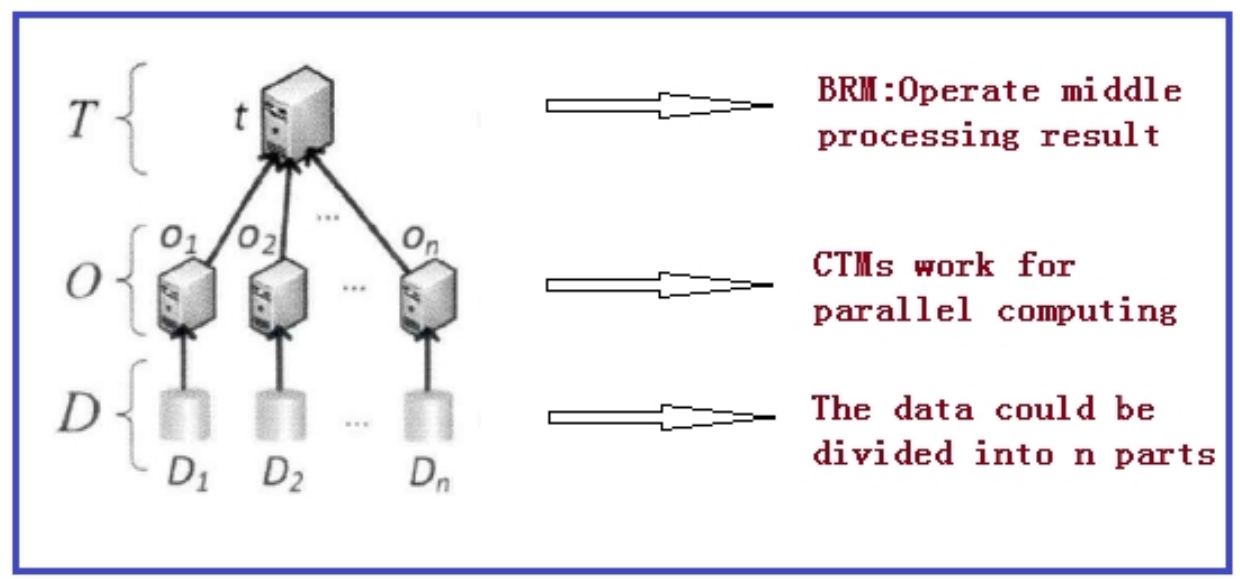

Fig 3. The composition of the parallel computing module

As shown in Fig 3,BRM is responsible for decomposing and integrating the behavior recognition task. The CTMs in the same group work for parallel computing, at the same time, the data is divided into $\mathrm{n}$ parts for CTM. BRM employ a distributed SVM classifier to classify moving targets behaviors. The solution could improve calculate ability and decrease the time for action module training.

\section{Feature matching and collaborative tracking}

$\mathrm{BRM}$ are responsible for recognition the behavior of moving target in some important scene using the algorithm that combine action library matching with moving trajectory detection [10]. The part is not the key point of the paper, but feature extraction would be process when BRM detect a suspicious behavior so as to CTM performance collaborative tracking.

An improved color histogram algorithm is proposed to extract target feature, which utilize the multi nodes characteristic of WSN. Fig4 is the sketch map of the method, feature of collaborative tracking target extract by different monitors and calculate the average value as the standard feature. In this way, feature error from different detection angle and illumination diversity could be decrease, so as to increase the veracity of feature match.

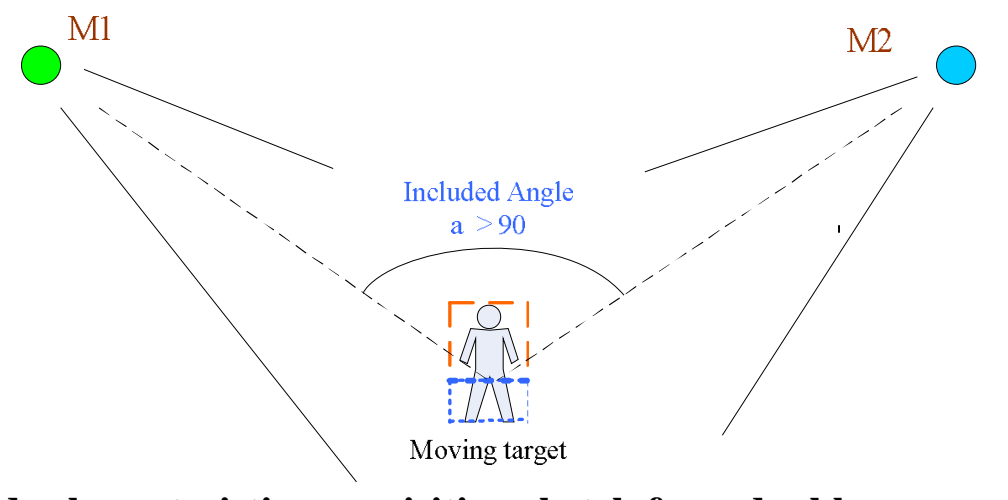

Fig 4. Node characteristics acquisition sketch from double perspectives

As shown in Fig4, M1 and M2 for two monitor nodes, generally choose the included angle is greater than 90 degrees, each point to extract the top and bottom color information of moving targets, then two monitor nodes on a weighted average of the characteristics of the same parts, features the average expression of target is obtained. We hope to get the higher similarity of two target color models, so the weight of particle must be higher. Bhattacharyya distance [11] can be used to give each particle value, $\sigma$ is the variance of Gausses distribution of color information. 


$$
\pi^{(n)}=\frac{1}{\sqrt{2 \pi} \sigma} e^{-\frac{d^{2}}{2 \sigma^{2}}}=\frac{1}{\sqrt{2 \pi} \sigma} e^{-\frac{\left(1-\rho\left[p_{s}(n), q\right]\right)}{2 \sigma^{2}}}
$$

Finally, considering the difficulties about moving target tracking to single monitor, for example, illumination change or shelter from, our solution revise the tracking result according to relevancy property $\alpha(\mathrm{x}, \mathrm{x}, \mathrm{y}), \mathrm{x}(\mathrm{x}, \mathrm{y}))$ and time difference $T(\mathrm{x}(\mathrm{x}, \mathrm{y}), \mathrm{x}(\mathrm{x}, \mathrm{y}))$. Relevancy property present whether the monitor $\mathrm{i}$ and $\mathrm{j}$ is connected. Time difference is the reference time when the target move from monitor i to monitor $\mathrm{j}$. The relevancy property and time difference are focus on static sensor monitors.

\section{Experiment and results}

To test the performance of WSN for moving target collaborative tracking described in this paper, a prototype system is developed to collaborative tracking suspicious target, using the development platform for VS2010 and computer vision library OPENCV, running environment of the system are as follows: Intel Xeon CPU, 64G RAM, and windows operating system.

This experiment in WSN by 20 distributions in different outdoor scene of monitoring points and a background management PC, through the background management software can see the WSN suspicious target collaborative tracking situation.In order to verify the algorithm described in this paper, the efficiency of abnormal body collaborative tracking, we from two aspects of detection accuracy and tracking efficiency made compared with similar algorithms, statistical results refer to Table 1:

Table 1. The result of statistics analysis for different algorithms

\begin{tabular}{|l|c|c|}
\hline \multicolumn{1}{|c|}{ Solutions } & Detection accuracy & Tracking accuracy \\
\hline Yan Zhou,etc [2] & $82.7 \%$ & $77.5 \%$ \\
\hline Cai Zi-xing,etc [4] & $78.9 \%$ & $81.6 \%$ \\
\hline Yuan Yuan [5] & $79.3 \%$ & $84.2 \%$ \\
\hline Our solution & $84.6 \%$ & $86.5 \%$ \\
\hline
\end{tabular}

We can see from the above results, Yan Zhou, etc used in a quantized measurement fusion framework is focus on the energy and bandwidth scheduling strategy is more prominent in the target detection precision, while in the target tracking accuracy is not ideal. And Cai Zi-xing, etc put forward a dynamic cluster member selection method for multi-target tracking in WSN scheme has better target tracking accuracy, but is not ideal in terms of target detection. Yuan Yuan proposed a visual object tracking method based on backward model validation is also the same problem. Thus it can be seen that described in this article, the target detection precision and the performance of synergy has better tracking performance.

\section{Conclusion and future works}

In this paper, we proposed a novel solution which utilizes the advantage of WSN to improve the feature extraction scheduling for moving human collaborative tracking. At first, WSN optimization deployment: divide the monitors in WSN into the two types: Behavior Recognition Monitors and Collaborative Tracking Monitors, and settle all the monitors utilizing FCM algorithm into many groups. Secondly, parallel detection and behavior recognition to get the collaborative tracking target. Finally, a multi-points feature extraction scheme for WSN monitors to collaborative track the suspicious target. After achieved the solution, we also compare our algorithm with three exist algorithms, the statistics result show that our scheme has a better detection accuracy and tracking performance. 


\section{Acknowledgement}

This work was supported by The National Natural Science Funds (Num: 61173091) in China.

\section{Reference}

[1] Xiaoqing Hu, Ming Bao, Yu-Hen $\mathrm{Hu}$, and Bugong Xu. Energy Balanced Scheduling for Target Tracking with Distance-Dependent Measurement Noise in a WSN. International Journal of Distributed Sensor Networks. Vol.2013, ID179623.

[2] Yan Zhou, Dongli Wang, Tingrui Pei, and Yonghong Lan. Energy-Efficient Target Tracking in Wireless Sensor Networks: A Quantized Measurement Fusion Framework. International Journal of Distributed Sensor Networks. Vol.2014. ID682032.

[3] Yanmin Zhu, Zhenzhen Tian, and Jiadi Yu. EMP: Exploiting Mobility Patterns for Collaborative Localization in Sparse Mobile Networks. International Journal of Distributed Sensor Networks. Vol.2014, ID370364.

[4] CAI Zi-xing, WEN Sha, LIU Li-jue. Dynamic cluster member selection method for multi-target tracking in WSN, J.Cent.South Univ. (2014)21:636-645.

[5] Yuan Yuan, Sabu Emmanuel, Yuming Fang, and Weisi Lin. Visual object tracking method based on backward model validation, IEEE TRANSACTIONS ON CIRCUITS AND SYSTEMS FOR VIDEO TECHNOLOGY,VOL.24, NO.11.2014.

[6] Oualid Demigha, Walid-Khaled Hidouci, Toufik Ahmed. On Energy Efficiency in Collaborative Target Tracking in Wireless Sensor Network: A Review. IEEE COMMUNICATIONS SURVEYS \& TUTORIALS,THIRD QUARTER 2013,VOL.15,NO.3,1210-1222.

[7] Jiang Lun, Ding Hua-fu. Improvement of the Fuzzy C-Means Clustering Algorithm,Computer \& Digital Engineering.Vol.38 No.24,p4-6,14.

[8] HAO Hong-xing, ZHU Yu-quan, CHEN Geng. A Hybrid Dynamic Clustering Algorithm based on Partition and Hierarchical Clustering. Computer Application Research, Jan.2011.vol.28 No.1 p51-53.

[9] Huai Y, Lee R, Zhang S, et al. DOT: a matrix model for analyzing, optimizing and deploying software for big data analytics in distributed systems. Proceedings of the 2nd ACM Symposium on Cloud Computing. ACM, 2011.

[10] Jing XIONG, ZhiJing LIU, HongMin XUE,etc. A Human Motion Recognition and Collaborative Tracking Algorithm. Journal of Computational Information System. 2015. No.4.

[11] I.Boulanouar, A.Rachedi, S.Lohier, G.Roussel, "Energy-Aware Object Tracking Algorithm using Heterogeneous Wireless Sensor Networks"IFIP Wireless Days Conference, Niagara Falls, Ontario, Canada.October, 2011 